\title{
Near-Capacity FEC Codes for Non-Regenerative
}

\section{MIMO-Aided Relays}

\author{
Soon Xin $\mathrm{Ng}^{1}$, Senior Member, IEEE, Wei Liu ${ }^{2}$, Member, IEEE, \\ Jian-Dong $\mathrm{Li}^{2}$, Senior Member, IEEE and Lajos Hanzo ${ }^{1}$ Fellow, IEEE \\ ${ }^{1}$ School of Electronics and Computer Science, University of Southampton, SO17 1BJ, United Kingdom. \\ Email: $\{$ sxn,lh\}@ecs.soton.ac.uk, http://www-mobile.ecs.soton.ac.uk \\ ${ }^{2}$ Broadband Wireless Communications Laboratory, Information Science Institute, State Key Laboratory of ISN, \\ Xidian University, Xi'an, China, 710071. \\ Email: \{liuweixd,jdli\}@mail.xidian.edu.cn, http://www.xidian.edu.cn/English/research_institutions/state_key_laboratories/
}

\begin{abstract}
In this contribution, we derive the Discrete-input Continuous-output Memoryless Channel (DCMC) capacity of the nonregenerative Multiple-Input Multiple-Output (MIMO) relay channel, when the source-to-destination link is inferior and hence considered absent. We design near-capacity Forward Error Correction (FEC) codes for approaching this capacity limit. It is shown that our design is capable of approaching the DCMC capacity within $0.4 \mathrm{~dB}$, when communicating over uncorrelated Raleigh fading channels, where the source node, relay node and destination node are equipped with two antennas each.
\end{abstract}

Index Terms - Cooperative Communications, MIMO, non-regenerative relay, Amplify-and-Forward, near-capacity codes, channel capacity.

\section{INTRODUCTION}

In cooperative communications [1]-[3] each mobile unit may collaborate with a partner or even several partners for the sake of reliably transmitting its own information and that of its partners. More specifically, a Source Node ( $\mathrm{SN}$ ) can transmit its signal to a Destination Node (DN) via a Relay Node (RN). Furthermore, it can also increase the energy-efficiency of the system due to the reduced path-loss experienced at the cooperative RN. Hence, cooperative systems exhibit an increased capacity, transmission reliability and energy efficiency. Due to the high capacity potential of the Multiple-Input Multiple-Output (MIMO) channels [4], [5], MIMOaided relaying techniques have been intensively investigated [6][8]. More specifically, a Decode-and-Forward (DF) based regenerative MIMO-aided relay was considered in [6], while its Amplifyand-Forward (AF) based non-regenerative counterpart was designed in [7]. The non-regenerative MIMO-aided relay system exhibits a lower complexity as well as a higher information-security [7], since the source signals are not regenerated at the relay. The optimum non-regenerative MIMO-aided relay matrix, which disregarded the direct Source-to-Destination (SD) link, has been designed in [7] for maximizing the achievable system capacity. However, the optimum non-regenerative MIMO-aided relay matrix is still unknown for the scenario when the direct SD link is available. Furthermore, the optimum coding and modulation schemes at the SN in the presence of a non-regenerative MIMO-aided relay is also an open research problem [7]. Moreover, although the corresponding Continuousinput Continuous-output Memoryless Channel (CCMC) capacity was derived in [7], the more practical modulation-dependent Discreteinput Continuous-output Memoryless Channel (DCMC) capacity has

The financial support from the RCUK for the UK-China Science Bridge Project: R\&D on (B)4G Wireless Mobile Communications, as well as from the National Basic Research Program of China (973 Program)(2009CB320404), the Program for Changiiang Scholars and Innovative Research Team in University (PCSIRT) (IRT0852), the ISN1003002, the Innovation Fund for returned overseas Scholars of Xidian University (64101879) and the China 111 Project (B08038) is gratefully acknowledged. The financial support from the European Union's Seventh Framework Programme under the auspices of the CONCERTO project (288502) is also gratefully acknowledged. not been quantified.

Against this background, in this contribution we first derive the modulation-dependent DCMC capacity formula for the nonregenerative MIMO-aided relay system when the direct SD link is absent, because the corresponding optimum non-regenerative MIMOaided relay matrix has been found in [7]. Furthermore, the role of the relay becomes significantly more important when the direct SD link is weak or absent. Then, we design near-capacity Forward Error Correction (FEC) codes to approach this limit. The proposed coded cooperative communication scheme is capable of reducing the transmit power, hence increasing the attainable energy efficiency. It can also assists in increasing the coverage area of a base station for improving the cell-edge performance. On one hand, the employment of multiple antennas at the RN increases the overall throughput of the system. On the other hand, the employment of powerful coding and modulation schemes enhance the system's resilience to channel fading. We aim for jointly designing the coding, modulation and multi-antenna based cooperative nodes for achieving an increased throughput, integrity and energy efficiency.

\section{SySTEM MODEL}

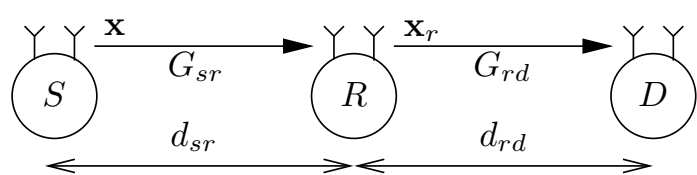

Fig. 1. Schematic of a two-hop relay-aided system, where $d_{a b}$ and $G_{a b}$ denote the geographical distance and the geometrical gain, respectively, between node $a$ and node $b$.

The schematic of our two-hop relay-aided system is shown in Fig. 1, where the SN $(S)$ transmits a frame of coded and modulated symbols $\{\mathbf{x}\}$ to the $\mathrm{RN}(R)$ during the first transmission period. Then, the RN amplifies and forwards the received signals to the DN $(D)$ in the form of $\left\{\mathbf{x}_{r}\right\}$ during the second transmission period. The communication links seen in Fig. 1 are subject to both long-term path loss as well as to short-term uncorrelated Rayleigh fading. We consider a free-space path loss model where the geometrical-gain experienced by the Source-to-Relay (SR) link as well as the Relayto-Destination (RD) link with respect to the SD link can be computed as [3]:

$$
G_{s r}=\left(\frac{d_{s d}}{d_{s r}}\right)^{2} \quad \text { and } \quad G_{r d}=\left(\frac{d_{s d}}{d_{r d}}\right)^{2}
$$

respectively, where $d_{a b}$ denotes the geographical distance between nodes $a$ and $b$. Without loss of generality, we assume that the RN is located at the mid-point between the SN and the DN, where we have $G_{s r}=G_{r d}=4$. 
We assume that the $\mathrm{SN}, \mathrm{RN}$ and $\mathrm{DN}$ are equipped with $L$ antennas. The $(L \times 1)$-element signal vector received at the $\mathrm{RN}$ during the first transmission period can be written as:

$$
\mathbf{y}_{r}=\sqrt{G_{s r}} \mathbf{H}_{s r} \mathbf{x}+\mathbf{n}_{r}
$$

where $\mathbf{x}$ is the $(L \times 1)$-element signal vector transmitted from the $\mathrm{SN}$, $\mathbf{H}_{s r}$ is the $(L \times L)$-element MIMO Rayleigh fading channel matrix between the $\mathrm{SN}$ and the RN, while $\mathbf{n}_{r} \sim \mathcal{C} \mathcal{N}\left(0,2 \sigma_{1}^{2} I_{L}\right)$ is the zeromean $(L \times 1)$-element AWGN vector having a covariance matrix of $2 \sigma_{1}^{2} I_{L}$. Similarly, the $(L \times 1)$-element signal vector received at the DN during the second transmission period may be written as:

$$
\mathbf{y}=\sqrt{G_{r d}} \mathbf{H}_{r d} \mathbf{x}_{r}+\mathbf{n}_{d},
$$

where $\mathbf{x}_{r}$ is the $(L \times 1)$-element signal vector transmitted from the $\mathrm{RN}, \mathbf{H}_{r d}$ is the $(L \times L)$-element MIMO Rayleigh fading channel matrix between the RN and the DN, while $\mathbf{n}_{d} \sim \mathcal{C N}\left(0,2 \sigma_{2}^{2} I_{L}\right)$ is the zero-mean $(L \times 1)$-element AWGN vector having a covariance matrix of $2 \sigma_{2}^{2} I_{L}$.

The transmit powers of the $\mathrm{SN}$ and $\mathrm{RN}$ are denoted as $P_{s}$ and $P_{r}$, respectively. Furthermore, we define the normalized Signal to Noise power Ratio (SNR) at the RN and DN as:

$$
\rho_{1}=\frac{P_{s}}{2 \sigma_{1}^{2} L} \text { and } \rho_{2}=\frac{P_{r}}{2 \sigma_{2}^{2} L},
$$

respectively. If $\mathbf{x}_{s}=\left[\begin{array}{lll}x_{s, 1} & \ldots & x_{s, L}\end{array}\right]^{T}$ is the symbol vector transmitted from the SN, the average SNR per receiver antenna at the RN is given by:

$$
\begin{aligned}
\mathrm{SNR}_{r} & =\frac{G_{s r}}{2 \sigma_{1}^{2}} \cdot \frac{\sum_{i=1}^{L} \sum_{j=1}^{L} \mathrm{E}\left\{\left|h_{s r, i, j}\right|^{2}\right\} \mathrm{E}\left\{\left|x_{s, j}\right|^{2}\right\}}{L} \\
& =\frac{G_{s r} P_{s}}{2 \sigma_{1}^{2}}=\rho_{1} L G_{s r}
\end{aligned}
$$

where $\mathrm{E}\left\{\left|h_{a b, i, j}\right|^{2}\right\}=1$ and $\mathrm{E}\left\{\left|x_{s, j}\right|^{2}\right\}=P_{s} / L$. For ease of analysis, we invoke the term transmit SNR as introduced in [2], [3], which is defined as the ratio of the power transmitted from the SN to the noise power encountered at the receiver of the $\mathrm{RN}$ :

$$
\mathrm{SNR}_{t}=\frac{1}{2 \sigma_{1}^{2}} \cdot \sum_{j=1}^{L} \mathrm{E}\left\{\left|x_{s, j}\right|^{2}\right\}=\frac{P_{s}}{2 \sigma_{1}^{2}} .
$$

Without the loss of generality, we consider the scenario of $\mathrm{SNR}_{t}=$ $P_{s} / 2 \sigma_{1}^{2}=P_{r} / 2 \sigma_{2}^{2}$ in this paper.

\section{OPTIMUM RELAYING MATRIX}

The performance of the AF based MIMO-aided relaying system can be significantly improved by jointly optimizing the transceivers at the SN, RN and DN. An optimal linear weighting matrix was designed for the RN in [7] for maximizing the achievable system capacity. For simplicity, let us define the equivalent $(L \times L)$-element channel matrix spanning from the $\mathrm{SN}$ to the $\mathrm{RN}$ as $\mathbf{H}_{1}=\sqrt{G_{s r}} \mathbf{H}_{s r}$ and that from the $\mathrm{RN}$ to the $\mathrm{DN}$ as $\mathbf{H}_{2}=\sqrt{G_{r d}} \mathbf{H}_{r d}$. Then the relayed signal can be written as:

$$
\mathbf{x}_{r}=\mathbf{F} \mathbf{y}_{r}=\mathbf{F H}_{1} \mathbf{x}+\mathbf{F n}_{r},
$$

where $\mathbf{F}$ is the relaying matrix. Consequently, the power constraint at the $\mathrm{RN}$ is given by:

$$
2 \sigma_{1}^{2} \operatorname{trace}\left\{\mathbf{F}\left(\mathbf{I}_{L}+\rho_{1} \mathbf{H}_{1} \mathbf{H}_{1}^{\dagger}\right)\right\} \leq P_{r},
$$

where $\mathbf{I}_{L}$ is an $(L \times L)$-element identity matrix and $(.)^{\dagger}$ denotes the hermitian transpose of (.). We further define the eigenvalue decompositions of the channel matrices as:

$$
\begin{aligned}
\mathbf{H}_{1}=\mathbf{U}_{1} \boldsymbol{\Lambda}_{1} \mathbf{V}_{1}^{\dagger} \quad ; \quad \mathbf{H}_{2}=\mathbf{U}_{2} \boldsymbol{\Lambda}_{2} \mathbf{V}_{2}^{\dagger} \\
\mathbf{H}_{1} \mathbf{H}_{1}^{\dagger}=\mathbf{U}_{1} \boldsymbol{\Sigma}_{1} \mathbf{U}_{1}^{\dagger} \quad ; \quad \mathbf{H}_{2}^{\dagger} \mathbf{H}_{2}=\mathbf{V}_{2} \boldsymbol{\Sigma}_{2} \mathbf{V}_{2}^{\dagger}
\end{aligned}
$$

where $\mathbf{U}_{1}$ and $\mathbf{V}_{2}$ are unitary matrices, while $\boldsymbol{\Lambda}_{1}, \boldsymbol{\Lambda}_{2}, \boldsymbol{\Sigma}_{1}=\boldsymbol{\Lambda}_{1} \boldsymbol{\Lambda}_{1}^{\dagger}$ and $\boldsymbol{\Sigma}_{2}=\boldsymbol{\Lambda}_{2}^{\dagger} \boldsymbol{\Lambda}_{2}$ are diagonal matrices. It was shown in [7] that the optimum non-regenerative MIMO-aided relaying matrix in the absence of the SD link is given by:

$$
\mathbf{F}=\mathbf{V}_{2} \boldsymbol{\Lambda}_{F} \mathbf{U}_{1}^{\dagger}
$$

where $\boldsymbol{\Lambda}_{F}$ is a diagonal matrix. Hence, the signal vector received at the DN given in Eq. (3) can be rewritten as:

$$
\begin{aligned}
\mathbf{y} & =\mathbf{H}_{2} \mathbf{F} \mathbf{H}_{1} \mathbf{x}+\mathbf{H}_{2} \mathbf{F} \mathbf{n}_{r}+\mathbf{n}_{d} \\
& =\mathbf{U}_{2} \boldsymbol{\Lambda}_{2} \boldsymbol{\Lambda}_{F} \boldsymbol{\Lambda}_{1} \mathbf{V}_{1}^{\dagger} \mathbf{x}+\mathbf{U}_{2} \boldsymbol{\Lambda}_{2} \boldsymbol{\Lambda}_{F} \mathbf{U}_{1}^{\dagger} \mathbf{n}_{r}+\mathbf{n}_{d} .
\end{aligned}
$$

The relay matrix derived in [7] is optimum for the case, where the $\mathrm{SN}$ does not know the channel matrices, but both the RN and the DN have accurate knowledge of the channel matrices $\mathbf{H}_{1}$ and $\mathbf{H}_{2}$. We found that the optimum relay matrix maximizing the CCMC capacity also maximizes the DCMC capacity in the low-SNR region. As shown in Fig. 2, the CCMC and DCMC capacities are identical when the SNR is lower than $-5 \mathrm{~dB}$. This is consistent with the findings in [9], [10] where the derivative of the mutual information between the channel input and channel output, with respect to the equivalent channel SNR equals to the corresponding Minimum Mean-Square Error (MMSE) formula, regardless of the input signal's statistics, when the SNR is low. At a capacity of 2 bits/symbol (bps), the SNR required for the DCMC is only $0.4 \mathrm{~dB}$ higher than that of the CCMC, when the optimum relaying matrix was employed by both systems. More specifically, the diagonal matrix $\boldsymbol{\Lambda}_{F}=\operatorname{diag}\left\{f_{1}, f_{2}, \ldots, f_{L}\right\}$ is related to the power allocation, which can be optimized similar to the water-filling method using the following equation [7]:

$f_{k}=\frac{\sigma_{2}}{\sigma_{1}} \sqrt{\frac{1}{2 \beta_{k}\left(1+\rho_{1} \alpha_{k}\right)}\left[\sqrt{\rho_{1}^{2} \alpha_{k}^{2}+4 \rho_{1} \alpha_{k} \beta_{k} \mu^{*}}-\rho_{1} \alpha_{k}-2\right]^{+}}$

where we have $[z]^{+}=\max (0, z)$, while $\alpha_{k}$ and $\beta_{k}$ are the eigenvalues of $\mathbf{H}_{1} \mathbf{H}_{1}^{\dagger}$ and $\mathbf{H}_{2}^{\dagger} \mathbf{H}_{2}$, respectively, arranged in descending order. The variable $\mu^{*}$ is a unique root of the following equation:

$g(\mu)=\sum_{k=1}^{L} \frac{1}{2 \beta_{k}}\left[\sqrt{\rho_{1}^{2} \alpha_{k}^{2}+4 \rho_{1} \alpha_{k} \beta_{k} \mu^{*}}-\rho_{1} \alpha_{k}-2\right]^{+}-\rho_{2} L$.

We compute the root $\mu^{*}$ using the bisection based root-finding algorithm.

As a benchmark, we also consider the so-called 'naive' scheme as introduced in [7], where the MIMO-aided relay invokes low complexity normalization of the received signal for meeting the power-constraint of Eq. (9). The corresponding MIMO-aided relay matrix is given by:

$$
\mathbf{F}=\mathbf{I}_{L} \cdot \frac{\sigma_{2}}{\sigma_{1}} \sqrt{\frac{\rho_{2} L}{\operatorname{trace}\left\{\mathbf{I}_{L}+\rho_{1} \mathbf{H}_{1} \mathbf{H}_{1}^{\dagger}\right\}}},
$$

which does not require the knowledge of $\mathbf{H}_{2}$.

\section{DCMC's Symbol TRANSITION PROBABILITy}

However, without the knowledge of $\mathbf{H}_{1}$ at the $\mathrm{SN}$ it is not possible to convert the MIMO relaying channel into parallel sub-channels. In our more practical scenario, where the knowledge of $\mathbf{H}_{1}$ is unavailable at the $\mathrm{SN}$, we have to jointly detect all $L$ source symbols in $\mathbf{x}=\left[\begin{array}{lll}x_{1} & \ldots & x_{L}\end{array}\right]^{T}$ using Maximum Likelihood (ML) detection. More specifically, we can rewrite Eq. (13) as:

$$
\mathbf{y}=\mathbf{H} \mathbf{x}+\mathbf{V} \mathbf{n}_{r}+\mathbf{n}_{d}=\mathbf{H} \mathbf{x}+\mathbf{n},
$$

where $\mathbf{H}=\mathbf{H}_{2} \mathbf{F} \mathbf{H}_{1}$ is the equivalent $(L \times L)$-element MIMO relay channel, while $\mathbf{V}=\mathbf{H}_{2} \mathbf{F}$ and $\mathbf{n}=\mathbf{V} \mathbf{n}_{r}+\mathbf{n}_{d}$ is the equivalent noise 
vector, which has a $(L \times L)$-element covariance matrix of:

$$
\mathbf{R}=2 \sigma_{2}^{2}\left(\frac{\sigma_{1}^{2}}{\sigma_{2}^{2}} \mathbf{H}_{2} \mathbf{F} \mathbf{F}^{\dagger} \mathbf{H}_{2}^{\dagger}+\mathbf{I}_{L}\right)
$$

where the $L$ noise components in $\mathbf{n}$ are correlated because $\mathbf{R}$ is a non-diagonal matrix. It is possible to whiten the noise vector $\mathbf{n}$ upon dividing both sides of Eq. (18) by $\mathbf{R}$. However, we can still reliably detect the signals even without the noise whitening process, as long as the variance of each noise component is accurately computed. More specifically, the received signal vector of Eq. (18) may also be represented as:

$$
\left[\begin{array}{c}
y_{1} \\
\vdots \\
y_{L}
\end{array}\right]=\left[\begin{array}{c}
\mathbf{h}_{1} \\
\vdots \\
\mathbf{h}_{L}
\end{array}\right] \mathbf{x}+\left[\begin{array}{c}
n_{1} \\
\vdots \\
n_{L}
\end{array}\right]
$$

where $\mathbf{h}_{i}$ is the $i$ th row vector of the $(L \times L)$-element matrix $\mathbf{H}$ and $n_{i}$ is the $i$ th element of the $(L \times 1)$-element noise vector $\mathbf{n}$. It may be readily shown that the effective noise variance for the $i$ th noise component $n_{i}$ is given by:

$$
N_{0, i}=2\left(\left\|\mathbf{v}_{i}\right\|^{2} \sigma_{1}^{2}+\sigma_{2}^{2}\right),
$$

where $\mathbf{v}_{i}$ is the $i$ th row vector of the $(L \times L)$-element matrix $\mathbf{V}$. Since $\mathbf{v}_{i}$ is a constant during a symbol period, each of the noise component is still an AWGN process. However, each $n_{i}$ has a different variance, because we have $\left\|\mathbf{v}_{i}\right\| \neq\left\|\mathbf{v}_{j}\right\|$ for $i \neq j$ and $i, j \in\{1, \ldots, L\}$. Note furthermore that $N_{0, i}=\left\|\operatorname{diag}_{i}\{\mathbf{R}\}\right\|$, where $\operatorname{diag}_{i}\{\mathbf{R}\}$ is the $i$ th diagonal element of the covariance matrix in Eq. (19), because the covariance of $n_{i}$ with itself equals its variance. Finally, based on Eqs. (20) and (21), the DCMC's symbol transition probability of receiving $\mathbf{y}$ at the $\mathrm{DN}$, given that $\mathbf{x}^{(m)}$ was transmitted from the $\mathrm{SN}$, may be computed by the MIMO demapper of the DN as:

$$
P\left(\mathbf{y} \mid \mathbf{x}^{(m)}\right)=\frac{1}{\prod_{j=1}^{L} \pi N_{0, j}} \exp \left(\sum_{i=1}^{L}-\frac{\left\|y_{i}-\mathbf{h}_{i} \mathbf{x}^{(m)}\right\|^{2}}{N_{0, i}}\right),
$$

where $\mathbf{x}^{(m)}=\left[x_{1}^{(m)} \ldots x_{L}^{(m)}\right]^{T}$ is the $m$ th combination from the set of $M^{L}$ possible symbol combinations, when an $M$-ary PSK/QAM modulation scheme is employed at the SN equipped with $L$ transmit antennas.

\section{Channel Capacity}

The CCMC capacity of the non-regenerative MIMO-aided relaying system dispensing the SD link was derived in [7] as:

$$
C_{0}=\log _{2}\left\|\mathbf{I}_{L}+\rho_{1} \mathbf{H}_{1} \mathbf{H}_{1}^{\dagger}-\rho_{1} \mathbf{H}_{1} \mathbf{H}_{1}^{\dagger} \mathbf{S}^{-1}\right\|,
$$

and

$$
\mathbf{S}=\mathbf{I}_{L}+\frac{\sigma_{1}^{2}}{\sigma_{2}^{2}} \mathbf{F}^{\dagger} \mathbf{H}_{2}^{\dagger} \mathbf{H}_{2} \mathbf{F}
$$

The DCMC capacity of the non-regenerative MIMO-aided relaying system of Eq. (18) may be derived as:

$$
\begin{aligned}
C_{\star}= & \max _{p(\mathbf{x})} \sum_{m=1}^{M^{L}} \int_{\mathbf{y}} P\left(\mathbf{y} \mid \mathbf{x}^{(m)}\right) P\left(\mathbf{x}^{(m)}\right) . \\
& \log _{2}\left(\frac{P\left(\mathbf{y} \mid \mathbf{x}^{(m)}\right)}{\sum_{n=1}^{M} P\left(\mathbf{y} \mid \mathbf{x}^{(n)}\right) P\left(\mathbf{x}^{(n)}\right)}\right) d \mathbf{y},
\end{aligned}
$$

where $P\left(\mathbf{y} \mid \mathbf{x}^{(m)}\right)$ is the DCMC's symbol transition probability given in Eq. (22) and the right hand side of Eq. (25) is maximized, when we have $P\left(\mathbf{x}^{(m)}\right)=1 / M^{L}$ for $m \in\left\{1, \ldots, M^{L}\right\}$. Then, Eq. (25) can be rewritten as:

$$
C_{\star}=\log _{2}\left(M^{L}\right)-\frac{1}{M^{L}} \sum_{m=1}^{M^{L}} \mathrm{E}\left[\log _{2} \sum_{n=1}^{M^{L}} \exp \left(\Psi_{m, n}\right) \mid \mathbf{x}^{(m)}\right],
$$

where $\mathrm{E}\left[f() \mid. \mathbf{x}^{(m)}\right]$ is the expectation of the function $f($.$) condi-$ tioned on $\mathbf{x}^{(m)}$, which is evaluated for different realizations of the equivalent channel matrix $\mathbf{H}$ and the equivalent noise vector $\mathbf{n}$. Furthermore, the exponential term $\Psi_{m, n}$ can be shown to be:

$$
\Psi_{m, n}=\sum_{i=1}^{L}-\frac{\left\|\mathbf{h}_{i}\left(\mathbf{x}^{(m)}-\mathbf{x}^{(n)}\right)+n_{i}\right\|^{2}+\left\|n_{i}\right\|^{2}}{N_{0, i}} .
$$

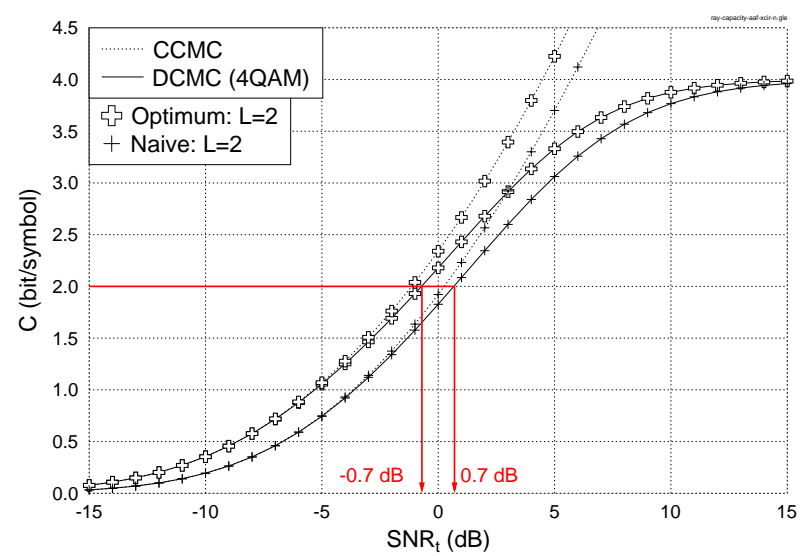

Fig. 2. The CCMC and 4QAM-based DCMC capacity curves of the non-regenerative MIMO-aided relay system when communicating over uncorrelated Rayleigh fading channels. The geometrical gains are given by $G_{s r}=G_{r d}=4$ and each of the $\mathrm{SN}, \mathrm{RN}$ and DN is equipped with $L=2$ antennas.

The CCMC and the 4QAM-based DCMC capacity curves of the non-regenerative MIMO-aided relaying systems, which employ both the optimum and naive relay matrices, are shown in Fig. 2, when communicating over uncorrelated Rayleigh fading channels. The geometrical gains are given by $G_{s r}=G_{r d}=4$ and each node is equipped with $L=2$ antennas. Similar to the conventional MIMO channel, the DCMC capacity and the CCMC capacity curves of the non-regenerative MIMO-aided relaying schemes are close to each other in the low-SNR region. Naturally, in the high-SNR region, the DCMC capacity curves will converge to the asymptotic limit of $\log _{2}\left(M^{L}\right)=4$ bits/symbol (bps).

As shown in Fig. 2, if a half-rate channel code is employed at the $\mathrm{SN}$, the minimum transmit SNR values required for achieving a DCMC capacity of 2 bps are $-0.7 \mathrm{~dB}$ and $0.7 \mathrm{~dB}$ for the optimum and the naive schemes, respectively. The transmit SNR values necessitated for attaining a CCMC capacity of 2 bps are $-1.1 \mathrm{~dB}$ and $0.3 \mathrm{~dB}$, respectively, which are only $0.4 \mathrm{~dB}$ better than those of their DCMC counterparts.

\section{TRANSCEIVER Design}

In this section we will design FEC codes to assist the nonregenerative MIMO-aided relaying system to approach the DCMC capacity with the aid of EXtrinsic Information Transfer (EXIT) charts [11], [12]. We will employ symbol-based inner recursive Unity Rate Code (URC) having a generator polynomial of $G(D)=$ $\frac{1}{1+D}$ [12] before the MIMO mapper as well as a bit-based outer Irregular Convolutional Code (IRCC) [13], [14] at the SN. More specifically, the general schematic of the non-regenerative MIMOaided relay transceiver is depicted in Fig. 3, where the source bits $\left\{u_{1}\right\}$ are encoded by an IRCC encoder. The IRCC-encoded bits $\left\{v_{1}\right\}$ 


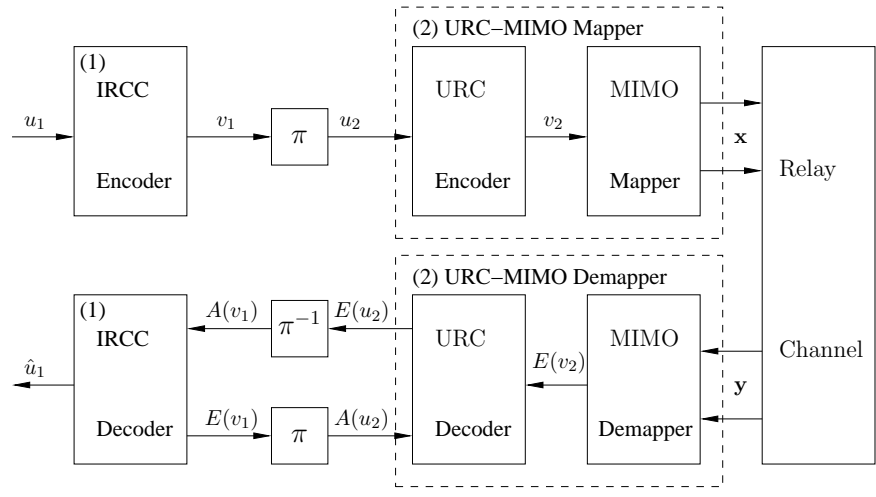

Fig. 3. The schematic of the non-regenerative MIMO-aided relay transceiver

of Fig. 3 are then interleaved for generating the stream $\left\{u_{2}\right\}$, and fed to the URC encoder ${ }^{1}$ for yielding a coded symbol sequence denoted as $\left\{v_{2}\right\}$. Then the MIMO mapper will map the symbol stream $\left\{v_{2}\right\}$ into the MIMO symbol vectors $\{\mathbf{x}\}$ for transmission over the relay channel. The relay's received signal vector $\mathbf{y}$ of Fig. 3 is modeled by Eq. (18). The notations $A(b)$ and $E(b)$ denote the a apriori and extrinsic probabilities of $b$, where $b \in\left\{v_{1}, u_{2} . v_{2}\right\}$. Iterative decoding between the IRCC decoder and URC decoder will be invoked at the DN for exchanging extrinsic probabilities of $u_{2}$ and $v_{1}$. The aim of the proposed transceiver design is to ensure that iterative decoding convergence is achieved at the lowest possible SNR, as facilitated by the corresponding relay channel capacity.

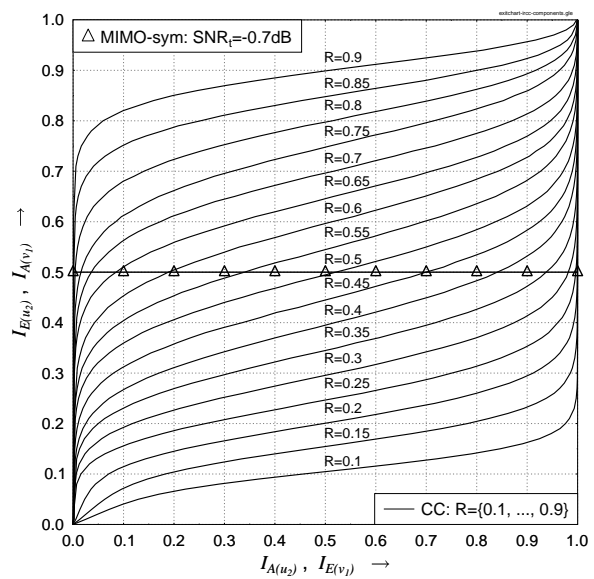

Fig. 4. The EXIT curves of $17 \mathrm{CC}$ decoders having a coding rate from 0.1 to 0.9 , as well as the normalized EXIT curve of the MIMO-sym demapper.

Let us consider the 4QAM $(M=4)$ based $L=2$ scenario. When the number of bits per $v_{2}$ symbol equals $l_{v}=4$, each of the MIMO symbol $\mathrm{x}$ corresponds to one $v_{2}$ symbol, as specified by the MIMO mapping of $\mathbf{x}=\mu\left(v_{2}\right)$. We term this mapper as the MIMO-sym mapper.The extrinsic probability $E\left(v_{2}\right)$ can then be computed as:

$$
E\left(v_{2}\right)=P(\mathbf{x} \mid \mathbf{y}) / P(\mathbf{x})=P(\mathbf{y} \mid \mathbf{x}) / P(\mathbf{y}) \approx P(\mathbf{y} \mid \mathbf{x}),
$$

where $P(\mathbf{y} \mid \mathbf{x})$ is given by Eq. (22) and $P(\mathbf{y})$ is a constant that may be ignored. The mutual information between $v_{2}$ and $E\left(v_{2}\right)$ can be computed as [15]:

$I_{E\left(v_{2}\right)}=\log _{2}\left(M^{L}\right)-\mathrm{E}\left[\sum_{m=1}^{M^{L}} E\left(v_{2}^{(m)}\right) \log _{2}\left(E\left(v_{2}^{(m)}\right)\right)\right]$.

\footnotetext{
${ }^{1}$ Note that before the URC encoding, the input bits $\left\{u_{2}\right\}$ are converted to symbols, where the bit-to-symbol convertor is assumed to be inside the URC Encoder block of Fig. 3.
}

We found that $I_{E\left(v_{2}\right)}=2 \mathrm{bps}$ at $\mathrm{SNR}_{t}=-0.7 \mathrm{~dB}$. If the URC is not used in Fig. 3, then we have $E\left(u_{2}\right)=E\left(v_{2}\right)$ and the corresponding normalized EXIT curve is plotted in Fig. 4, where $I_{A(.)}$ and $I_{E(.)}$ denote the mutual information corresponding to the a priori probability $A($.$) and extrinsic probability E($.$) [12], respectively. When the$ area of the whole EXIT chart is normalized to unity, the area under the EXIT curve of $I_{E\left(v_{2}\right)}$ becomes $A_{*}=I_{E\left(v_{2}\right)} / C_{*}^{\max }=0.5$ at $\mathrm{SNR}_{t}=-0.7 \mathrm{~dB}$, where $C_{*}^{\max }=\log _{2}\left(M^{L}\right)=4$ is the asymptotic DCMC capacity.

According to the so-called area property of the EXIT charts [13], [14], it can be shown that the area under the normalized EXIT curve of an inner decoder/demapper is related to the achievable DCMC capacity (namely $C_{*}$ in Eq. (26)) according to $A_{*}=C_{*} / C_{*}^{\max }$. Since, we have $C_{*}=I_{E\left(v_{2}\right)}=2 \mathrm{bps}$ at $\mathrm{SNR}_{t}=-0.7 \mathrm{~dB}$, we have shown empirically that the area property also holds true for the non-regenerative MIMO relay channel. We employ a symbol-based URC before the MIMO-sym mapper. More specifically, a $M^{L}=16$ state symbol-based URC having a generator polynomial of $G(D)=$ $\frac{1}{1+D}$ [12] can transform the horizontal EXIT curve to a slanted EXIT curve which becomes capable of reaching the top-right corner of the EXIT chart, as shown in Fig. 5. We found that the area under the slanted EXIT curve of the URC-MIMO-sym demapper equals that under the horizontal EXIT curve of the MIMO-sym demapper, when an A Posteriori Probability (APP) based decoder is used for the URC decoder. The mutual information between the URC's input bit $u_{2}$ and the extrinsic probability $E\left(u_{2}\right)$ at the output of the URC decoder may be characterized by its EXIT curve, which is characterized by the transfer function $T_{u_{2}}$ :

$$
I_{E\left(u_{2}\right)}=T_{u_{2}}\left[I_{A\left(u_{2}\right)}, I_{E\left(v_{2}\right)}\right]=T_{u_{2}}\left[I_{A\left(u_{2}\right)}, C_{*}\right] .
$$

On the other hand, the area under the inverted EXIT curve of an outer decoder equals to its coding rate $R_{*}$. We consider an IRCC that consists of $P=17$ memory-four Convolutional Codes (CCs) [13], [14], a total encoded sequence length of $N_{c}=240^{\prime} 000$ bits and an effective coding rate of $R_{*}=0.5$. The $k$ th subcode has a coding rate of $r_{k}$ and it encodes a fraction of $\alpha_{k} r_{k} N_{c}$ information bits to $\alpha_{k} N_{c}$ encoded bits. More specifically, $\alpha_{k}$ is the $k$ th IRCC weighting coefficient satisfying the following constraints [13], [14]:

$$
\sum_{k=1}^{P} \alpha_{k}=1, R_{*}=\sum_{k=1}^{P} \alpha_{k} r_{k}, \alpha_{k} \in[0,1], \forall k,
$$

which can be conveniently represented in the following matrix form:

$$
\begin{aligned}
{\left[\begin{array}{cccc}
1 & 1 & \ldots & 1 \\
r_{1} & r_{2} & \ldots & r_{P}
\end{array}\right]\left[\begin{array}{lll}
\alpha_{1} & \alpha_{2} \ldots & \alpha_{P}
\end{array}\right]^{T}=} & {\left[\begin{array}{c}
1 \\
R_{*}
\end{array}\right] } \\
\mathbf{C} \boldsymbol{\alpha} & =\mathbf{d} .
\end{aligned}
$$

The transfer function of the IRCC is given by:

$$
I_{E\left(v_{1}\right)}=T_{v_{1}}\left[I_{A\left(v_{1}\right)}\right]=\sum_{k=1}^{P} \alpha_{k} T_{v_{1}, k}\left[I_{A\left(v_{1}\right)}\right],
$$

where $T_{v_{1}, k}\left[I_{A\left(v_{1}\right)}\right]=I_{E\left(v_{1}\right), k}$ is the transfer function of the $k$ th subcode. More explicitly, the inverted EXIT curves of the $P=17$ subcodes having different coding rates ranging from 0.1 to 0.9 are shown in Fig. 4.

Based on these EXIT chart properties, a near capacity concatenated-coding scheme can be designed by matching the corresponding inner and outer decoder EXIT curves, so that a narrow but marginally open EXIT chart tunnel exists between them all the way to the $(x, y)=(1, y)$ point of Fig. 4 , where $x=I_{E\left(v_{1}\right)}=I_{A\left(u_{2}\right)}$ and $y=I_{A\left(v_{1}\right)}=I_{E\left(u_{2}\right)} \in\{0,1\}$. In other words, once the area properties are satisfied, our near-capacity code design becomes a curve-matching exercise. We employ the iterative method of [13], [14] in order to find the optimum solution of the IRCC weight vector 
Observe that it is not possible to match the horizontal EXIT curve of the MIMO-sym demapper shown in Fig. 4 using the 17 EXIT curves of the IRCC subcodes depicted in Fig. 4. However, it is possible to transform the horizontal EXIT curve to a slanted one by employing the symbol-based URC of Fig. 3 before the MIMO-sym mapper. More specifically, the slanted EXIT curve of the $M^{L}=16$ state symbol-based URC succeeds in reaching the top-right corner of the EXIT chart, as shown in Fig. 5. This slanted EXIT curve can now be matched using the 17 EXIT curves of the IRCC subcodes depicted in Fig. 4.

\section{RESUlTS AND DISCUSSIONS}

Based on the algorithm of Section VI, optimum IRCC schemes were designed for the MIMO-sym schemes employing both the optimum and naive relay matrices. The MIMO-sym Optimum scheme uses only six subcodes and its IRCC weight is given by $\boldsymbol{\alpha}=\left[0.249385 z^{3}+0.0449917 z^{4}+0.148072 z^{6}+0.185947 z^{9}+\right.$ $\left.0.201898 z^{13}+0.169739 z^{17}\right]$, where the exponent of the dummy variable $z$ denotes the index of the subcodes used in the IRCC. By contrasts, the MIMO-sym naive scheme requires eleven subcodes with an IRCC weight of $\boldsymbol{\alpha}=\left[0.184586 z^{3}+0.103116 z^{4}+0.122562 z^{6}+\right.$ $0.0107969 z^{7}+0.105622 z^{8}+0.0753062 z^{9}+0.0235839 z^{10}+$ $\left.0.0357434 z^{12}+0.177077 z^{13}+0.0140411 z^{16}+0.147571 z^{17}\right]$.

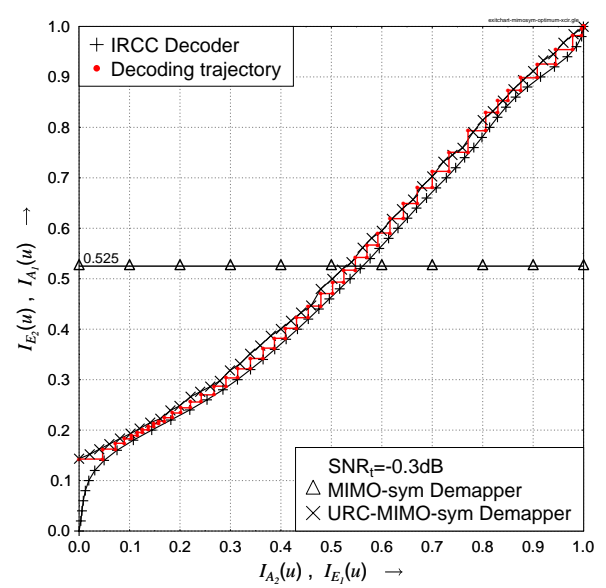

Fig. 5. The EXIT curves of the MIMO-symbol based optimum scheme.

The corresponding EXIT chart of the MIMO-sym based optimum scheme is shown in Fig. 5, where the outer decoder's IRCC EXIT curve perfectly matches the inner URC-MIMO-sym EXIT curve. As shown in Fig. 5, the simulation-based step-wise decoding trajectory succeeds in traversing through the narrow but marginally open EXIT tunnel to the top-right corner of its EXIT chart, when a sufficiently high number of decoding iterations are invoked between the URCMIMO-sym demapper and the IRCC decoder. This confirms that the simulations perfectly match the EXIT chart predictions. Furthermore, when $\mathrm{SNR}_{t}=-0.3 \mathrm{~dB}$, the areas under the EXIT curves of both the MIMO-sym and URC-MIMO-sym demappers are equal to 0.525. Since the area under the EXIT curve of the IRCC decoder equals $R_{*}=0.5$, the area within the narrow tunnel equals 0.025 . Hence, the proposed IRCC based URC-MIMO-sym scheme is only $0.025 C_{*}^{\max }=0.1 \mathrm{bps}$ away from the MIMO relay DCMC capacity. In terms of the SNR thresholds, it is only $-0.3-(-0.7)=0.4 \mathrm{~dB}$ and $-0.3-(-1.1)=0.8 \mathrm{~dB}$ away from the non-regenerative MIMO relay DCMC and CCMC capacities, respectively. The EXIT charts of the MIMO-sym naive scheme exhibit the same trends, but its EXIT chart tunnel only appears at $\mathrm{SNR}_{t}=1.2 \mathrm{~dB}$, which is $1.5 \mathrm{~dB}$ higher than that of the MIMO-sym optimum scheme.

\section{CONCLUSIONS}

We have derived the DCMC capacity of the non-regenerative MIMO-aided relaying system and found that the EXIT chart area properties are also valid for this system. We have studied both the optimum and naive relaying schemes and shown that the optimum relay matrix designed for the CCMC model is also optimum for the DCMC model at the low-SNR region. Then, near-capacity FEC codes operating at low-SNR region were designed to assist the non-regenerative MIMO-aided relay systems in approaching their relay channel capacities. Our proposed 16-state URC based optimum scheme is only $0.4 \mathrm{~dB}$ and $0.8 \mathrm{~dB}$ away from the corresponding DCMC and CCMC capacities, respectively.

\section{REFERENCES}

[1] A. Sendonaris, E. Erkip and B. Aazhang, "User cooperation diversity Part I: System description," IEEE Transactions on Communications, vol. 51(11), pp. 1927-1938, 2003.

[2] B. Zhao and M. C. Valenti, "Distributed turbo coded diversity for relay channel," IEE Electronics Letters, vol. 39, pp. 786-787, May 2003.

[3] H, Ochiai, P. Mitran and V. Tarokh, "Design and analysis of collaborative diversity protocols for wireless sensor networks," in Proceedings of IEEE VTC Fall, (Los Angeles, USA), pp. 4645 - 4649, 26-29 September 2004.

[4] E. Telatar, "Capacity of multi-antenna Gaussian channels," European Transactions on Telecommunication, vol. 10, pp. 585-595, Nov-Dec 1999.

[5] S. X. Ng and Hanzo, "On the MIMO channel capacity of multidimensional signal sets," IEEE Transactions on Vehicular Technology, vol. 55, pp. 528-536, March 2006.

[6] B. Wang, J. Zhang, and A. Host-Madsen, "On the capacity of MIMO relay channels," IEEE Transactions on Information Theory, vol. 51, pp. 29-43, Jan. 2005.

[7] X. Tang and Y. Hua, "Optimal design of non-regenerative MIMO wireless relays," IEEE Transactions on Wireless Communications, vol. 6 , pp. 1398-1407, April 2007.

[8] Y. Rong, X. Tang, and Y. Hua, "A unified framework for optimizing linear nonre-generative multicarrier MIMO relay communication systems," IEEE Transactions on Signal Processing, vol. 57, pp. 4837-4851, December 2009.

[9] A. Lozano, A. M. Tulino and S. Verdu, "Optimum power allocation for parallel Gaussian channels with arbitrary input distributions," IEEE Transactions on Information Theory, vol. 52, pp. 3033-3051, July 2006.

[10] D. Guo, S. Shamai and S. Verdu, "Mutual information and minimum mean-square error in Gaussian channels," IEEE Transactions on Information Theory, vol. 51, pp. 1261-1283, April 2005.

[11] S. ten Brink, "Convergence behaviour of iteratively decoded parallel concatenated codes," IEEE Transactions on Communications, vol. 49, pp. 1727-1737, October 2001.

[12] L. Hanzo, T. H. Liew, B. L. Yeap, R. Y. S. Tee and S. X. Ng, Turbo Coding, Turbo Equalisation and Space-Time Coding: EXIT-Chart-Aided Near-Capacity Designs for Wireless Channels, 2nd Edition. New York, USA: John Wiley IEEE Press, March 2011.

[13] M. Tüchler and J. Hagenauer, "EXIT charts of irregular codes," in Proceedings of Conference on Information Science and Systems, (Princeton University), pp. 465-490, 20-22 March 2002.

[14] M. Tüchler, "Design of serially concatenated systems depending on the block length," IEEE Transactions on Communications, vol. 52, pp. 209218, February 2004.

[15] J. Kliewer, S. X. Ng and L. Hanzo, "Efficient computation of EXIT functions for non-binary iterative decoding," IEEE Transactions on Communications, vol. 54, pp. 2133-2136, December 2006. 